Acta cryst. (2002). A58 (supplement), c35

\section{T CELL RECEPTOR-MHC RECOGNITION}

I.A. Wilson

The Scripps Research Institute Department Of Molecular Biology, Bcc206

10550 No. Torrey Pines Road La Jolla CA 92037 USA

The t-cell receptor (tcr) is a heterodimeric glycoprotein expressed on the surface of $t$ lymphocytes that plays a central role in the recognition of foreign antigens from microbial pathogens or transformed cells in the context of the major histocompatibility complex (mhc). The ability to discriminate between self and foreign antigens resides in the fine specificity of the tcr interaction with mhc. We have determined crystal structures of the murine class i tcr $2 \mathrm{c}$ bound to self and non-self agonist peptides, and compared them with other murine and human class i and class ii tcr-pmhc complexes. The tcrs all dock onto their respective pmhc in a similar, diagonal orientation, but with variable contacts that provide specificity to the bound peptide and individual mhe. Conformational changes in the central cdr loops of the tcr facilitate the dual recognition of self (syngeneic) and foreign (allogeneic) mhe complexes. These tcr-pmhc structures have begun to probe the molecular mechanisms of alloreactivity, cross-reactivity, graft rejection, positive and negative selection, and autoimmunity. The range of antigens presented by mhc or mhc-like molecules has recently been extended to include large bulged peptides, glycopeptides, lipids, and glycolipids. Thus, the tcr can interact with a much broader diversity of microbial and self-antigens than was previously anticipated. Thus, the adaptive immune systems can respond not only to tumor antigens or foreign proteins from microbial pathogens, but also to non-protein components from the cell walls of mycobacteria, such as tuberculosis and leprosy.

\section{Keywords: PROTEIN LIGAND INTERACTIONS, STRUCTURAL IMMUNOLOGY, CONFORMATIONAL CHANGES}

Acta Cryst. (2002). A58 (Supplement), C35

\section{STRUCTURAL BASIS FOR SITE-SPECIFIC RECOGNITION OF} NUCLEOSOMAL DNA

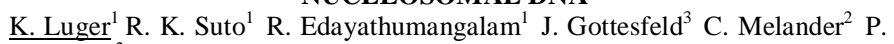
B. Dervan $^{2}$

${ }^{1}$ Colorado State University, Fort Collins, $\mathrm{CO}{ }^{2}$ California Institute of Technology, Pasadena, CA ${ }^{3}$ The Scripps Research Institute, San Diego, CA

The DNA of a single eukaryotic cell is over two meters in length, but compacts in the nucleus to nearly one millionth of this dimension as tight protein-DNA assemblies termed chromatin. At the first level, two tight superhelical turns of DNA are wrapped around an octamer of four histone proteins. This unit, the nucleosome core particle, represents the basic repeating unit in chromatin. Nucleosomal DNA is highly distorted and partially occluded from the solvent due to its tight interaction with the histone proteins.

The ability of DNA-binding proteins to recognize their cognate sites in chromatin is restricted by the structure and dynamics of nucleosomal DNA and by the translational and rotational positioning of the DNA with respect to the histone octamer. We use high-affinity sequence-specific pyrrole-imidazole polyamides as molecular probes to study DNA accessibility and flexibility in nucleosomes that contain a defined 146 base pair DNA sequence. We present the first high-resolution crystal structures of nucleosome core particles in complex with sequence-specific DNA binding ligands.

We show that while the overall structure of the nucleosome core particle remains unaffected by ligand binding, nucleosomal DNA undergoes significant structural changes that affect the ligand binding site and adjacent regions. In addition, these structures provide unprecedented insight into the mechanism by which structural changes are propagated from the end of the nucleosomal DNA over significant distance, and are thus highly relevant for our understanding of the mechanism of ATP-dependent chromatin remodeling factors and temperature-induced histone octamer translocation on the DNA.

Keywords: CHROMATIN NUCLEOSOME PYRROLE-IMIDAZOLE POLYAMIDE
Acta cryst. (2002). A58 (supplement), c35

CRYO ELECTRON MICROSCOPY SHOWS THAT MYOSIN SWITCH 1 OPENS ON STRONG BINDING TO ACTIN

K.C. Holmes I. Angert W. Jahn R.R. Schroeder

Max Planck Institute For Medical Research, 69120 Heidelberg Germany

The $50 \mathrm{k}$ domain of the myosin cross bridge is split into two subdomains (upper and lower) by the major cleft that connects the actin binding site with the nucleotide binding site. A number of lines of experimental evidence now point to the major cleft closing on strong binding of the cross bridge to actin. In particular, our high resolution (18 å) cryo em images using an energy filter to reduce inelastic scattering (eftem) show that most of the $50 \mathrm{k}$ upper domain has moved on strong binding to actin. Strategically placed loops permit this motion. However, the $50 \mathrm{k}$ upper domain carries the switch 1 element of the nucleotide-binding site so that the strong binding to actin will lead to a 5-6 movement of switch 1 . In fact strong binding to actin opens the nucleotidebinding pocket. This movement is quite distinct from the opening of switch 2 , which is caused by a $5^{\circ}$ rotation of the $50 \mathrm{k}$ lower domain with respect to the rest of the motor domain. While the opening/closing of switch 2 has been observed a number of times by $\mathrm{x}$-ray crystallography, the closing of the major cleft or opening of the nucleotide binding pocket has not been seen in isolated cross bridges. The movement of switch 1 during actin binding can account for the notable reciprocal relationship between actin binding affinity and nucleotide binding affinity.

Keywords: CRYO ELECTRONMICROSCOPY ACTIN MYOSIN

Acta Cryst. (2002). A58 (Supplement), C35

CRYSTAL STRUCTURE OF COLICIN E3: IMPLICATIONS FOR RIBOSOME INACTIVATION AND CELL ENTRY

M. Shoham $^{1}$ N. Wu ${ }^{1}$ S. Soelaiman ${ }^{1}$ K.R. Dickman ${ }^{1}$ A.P. Kosmidis ${ }^{1}$ C. Li $^{1}$ K. Jakes $^{2}$

${ }^{1}$ Department of Biochemistry, Case Western Reserve University, Cleveland, Ohio, USA ${ }^{2}$ Department of Physiology and Biophysics, Albert Einstein College of Medicine, Brox, NY, USA

Colicins kill e. Coli by a process that involves binding to a surface receptor, entering the cell and finally intoxicating it. The lethal action of colicin e 3 is a specific cleavage of the phosphodiester bond between a1493 and g1494 of 16s rrna in the ribosomal decoding a-site. Following this cleavage protein biosynthesis ceases and the cell dies. The producing cell is protected from the lethal action of colicin e3 by a tightly bound inhibitor, the immunity protein (ip). The crystal structure of colicin e 3 in a binary complex with ip reveals a yshaped molecule with the receptor-binding domain forming a 100 å long stalk and the two globular heads of the translocation domain $(\mathrm{t})$ and the catalytic domain (c) comprising the two arms. Active site residues in the rnase domain are $\mathrm{d} 510$, h513, e517 and r545. Ip is located between $\mathrm{t}$ and $\mathrm{c}$ with $83 \%$ of its surface buried by these interactions. Surprisingly, ip does not block the active site. The presence of ip prevents access of colicin e 3 to its huge substrate, the ribosome, either by steric hindrance or by charge repulsion of the acidic ip to negatively charged 16 s rrna.

\section{Keywords: RIBOSOME, RNASE, ANTIBIOTIC}

